Original article

\title{
Diagnostic accuracy of sonography in assessment of thyroid masses in comparison with pathology
}

\author{
Morteza Tahmasebi, Mohammad Reza Boostani Dezfouli, Mohammad Momen Gharibvand, Alireza Jahanshahi, \\ Neda Nikpour, Fakher Rahim
}

Ahvaz Jundishapur University of Medical Sciences, Ahvaz, Iran

Received 9 August 2015, Accepted 27 November 2015

(C) 2015, Tahmasebi M., Dezfouli M.R.B., Gharibvand M.M., Jahanshahi A., Nikpour N., Rahim F.

(C) 2015, Russian Open Medical Journal

\begin{abstract}
A thyroid nodule is a solid or cystic lump on the thyroid gland, which involves in the occurrences of various thyroid carcinomas with a notable clinical importance. The present study aimed to observe the diagnostic accuracy of ultrasound (US) in the assessment of thyroid masses compared to pathologic findings. This analytical, epidemiologic study was conducted on 307 patients that were referred to the radiology unit between November 2013 and March 2014 for fine needle aspiration (FNA) of suspected thyroid nodules. There was a significant correlation between echogenicity and pathologic results, which mean more hyper-echogenicity leads to more benign condition $(P=0.004)$. There was no significant correlation between the calcification and the marginal appearance with pathologic results $(P=0.270$, $\mathrm{P}=0.860$, respectively). There was a significant correlation between the length and width with pathologic results, which mean the shorter length and width lead to a more benign condition $(P=0.015, P=0.040$, respectively). Besides, there was no significant correlation between vascularity or the age with pathologic results $(P=0.450, P=0.930$, respectively). This study showed that US could give an incremental value to differentiate benign and malignancy thyroid nodule. Due to the high specificity and predicted values in the detection of benign cases, ultrasound findings can help to favor invasive procedures or follow up of thyroid nodules.
\end{abstract}

Keywords: thyroid nodules, fine needle aspiration, ultrasonography, pathology

Cite as Tahmasebi M, Dezfouli MRB, Gharibvand MM, Jahanshahi A, Nikpour N, Rahim F. Diagnostic accuracy of sonography in assessment of thyroid masses in comparison with pathology. Russian Open Medical Journal 2016; 5: e0103.

Correspondence to Mohammad Momen Gharibvand. Address: Department of Radiology, Golestan Hospital, Ahvaz Jundishapur University of Medical Sciences, Ahvaz, Iran. E-mail: mmmohamad93@yahoo.com

\section{Introduction}

A thyroid nodule is a solid or cystic lump on the thyroid gland. Thyroid nodule may be involved in various thyroid carcinomas with a noteworthy clinical importance [1]. The prevalence of thyroid nodules increased in an age-dependent manner, expanding to over $50 \%$ of the world's population [2]; while, further examination by ultrasonography (US) will detect additional nodules in $50 \%$ of people with solitary nodules detected by experienced physicians [3]. Biopsy examinations using fine needle aspiration (FNA) proved that around $5 \%$ of all nodules are cancerous [4]. Some studies show that 30 to $50 \%$ of the asymptomatic population will have at least one thyroid nodule found incidentally on US, which about 5$11 \%$ and even up to $30 \%$ of them can be malignant $[5,6]$.

US is the most broadly performed procedure in the thyroid gland screening. It has easy performance, cost effectiveness and sensitivity in determining the size and number of nodules. However, the great question is the role of ultrasonography in triage of thyroid nodules.

Many reports suggested a substantial overlap between the sonography findings in benign and malignant thyroid nodules, which such information may interpret as the vague concept of US in the detection of malignant nodules [7, 8]. So, different range of sensitivity and specificity of US for detecting malignant thyroid nodules are reported in various studies. The sensitivity and specificity of US in detecting palpable malignant thyroid tumors ranged from $80 \%$ to $100 \%$ and from $40 \%$ to $91 \%$, respectively.

However, assessing the accuracy of US diagnosis of nonpalpable benign and malignant nodules of the thyroid is promising and considering diverse findings from various studies, the present study aimed to observe the diagnostic accuracy of US in assessment of thyroid masses compared to pathologic findings.

\section{Material and Methods}

\section{Study Design and Population}

This analytical, epidemiologic study was conducted on 307 patients that were referred to the radiology unit between November 2013 and March 2014 for evaluation of suspected thyroid nodular disease. Then eventually their cytologic specimens (collected by FNA or surgery), were studied. The study was approved by the Ethical Committee of Ahvaz Jundishapur University of Medical sciences, and all patients signed the informed consent prior to enrollment.

Inclusion criteria: Patients with US detectable thyroid nodules in presence of normal levels of FT3, FT4, TSH, and candidate to FNA or surgery .

Exclusion criteria: History of previous surgery. 
Table 1. Baseline characteristics of studied group ( $n=\mathbf{3 0 7})$

\begin{tabular}{|c|c|c|}
\hline Baseline characteristics & Number & Percentage \\
\hline $\begin{array}{c}\text { Echogenicity } \\
\text { - Hypo } \\
\text { - Iso } \\
\text { - Hyper }\end{array}$ & $\begin{array}{c}101 \\
10 \\
196\end{array}$ & $\begin{array}{c}32.9 \\
3.3 \\
63.8\end{array}$ \\
\hline $\begin{array}{cl}\text { Calcification } \\
\text { - } & \text { Micro } \\
\text { - } & \text { Coarse } \\
\text { - } & \text { Non } \\
\end{array}$ & $\begin{array}{c}26 \\
2 \\
279\end{array}$ & $\begin{array}{c}8.5 \\
0.7 \\
90.8\end{array}$ \\
\hline $\begin{array}{l}\text { Margin appearance } \\
\text { - Specified } \\
\text { - Unknown }\end{array}$ & $\begin{array}{c}303 \\
4\end{array}$ & $\begin{array}{c}98.7 \\
1.3\end{array}$ \\
\hline $\begin{array}{l}\text { Length, } \mathrm{cm} \\
\text { - } \quad 1-10 \\
\text { - } \quad 11-20 \\
\text { - } \quad 21-30 \\
\text { - } \quad>30\end{array}$ & $\begin{array}{c}5 \\
157 \\
58 \\
35\end{array}$ & $\begin{array}{l}18.6 \\
51.1 \\
18.9 \\
11.4\end{array}$ \\
\hline 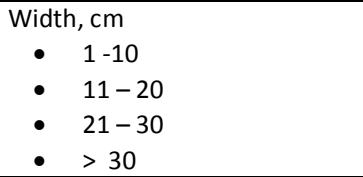 & $\begin{array}{c}140 \\
120 \\
39 \\
8\end{array}$ & $\begin{array}{c}45.6 \\
39.1 \\
12.7 \\
2.6\end{array}$ \\
\hline \begin{tabular}{cl}
\multicolumn{2}{l}{ Vascularity } \\
- & Decreased \\
- & Normal \\
- & Increased \\
\end{tabular} & $\begin{array}{c}52 \\
245 \\
10\end{array}$ & $\begin{array}{c}16.9 \\
79.8 \\
3.3\end{array}$ \\
\hline $\begin{array}{ll}\text { Age, years } \\
\begin{array}{ll}\text { - } & 20-29 \\
\text { - } & 30-39 \\
\text { - } & 40-49 \\
\text { - } & 50-59 \\
\text { - } & >60\end{array}\end{array}$ & $\begin{array}{c}29 \\
102 \\
100 \\
59 \\
17\end{array}$ & $\begin{array}{c}9.4 \\
33.2 \\
32.6 \\
19.2 \\
5.5\end{array}$ \\
\hline $\begin{array}{cl}\text { Pathologic report } \\
\text { - } & \text { Benign } \\
\text { - } & \text { Malignant } \\
\text { - } & \text { Unknown }\end{array}$ & $\begin{array}{c}287 \\
12 \\
8\end{array}$ & $\begin{array}{c}93.5 \\
3.9 \\
2.6\end{array}$ \\
\hline
\end{tabular}

\section{Methods}

The US findings and the final report, including benign or malignant nodule diagnosis were recorded. If there were indications for Thyroid US examination, patients were undergone FNA (US-guided FNA) or surgical treatment. Then, FNA results were compared with US findings and lastly sensitivity, specificity, positive predictive value and negative predicted value and accuracy were calculated .US findings were indicative for malignancy (marginal hyper-echogenicity, disappearing microcalcifications, size of more than $10 \mathrm{~mm}$, and vascularization of the nodule). For each nodule, sonographic images were reviewed by three radiology experts, and US characteristics were recorded. For each nodule, the following sonography characteristics were recorded: size, parenchymal composition, echogenicity, and marginal appearance. Size was recorded in three orthogonal dimensions. US images were acquired using a digital US imaging system Phillips HDI 5000 with a $12 \mathrm{MHz}$ linear transducer in the radiology department of Golestan hospital in Ahvaz, Iran.

The variables of interest were assessed according to the Phuttharak et al. protocol [9]. In brief, echogenicity of the thyroid nodule was measured by comparing echogenicity of the thyroid parenchyma and cervical muscle, and was classified as markedly hypoechoic, showing fairly more hypoechoic than adjacent cervical muscle, hypoechoic, showing relatively more hypoechoic than normal thyroid parenchyma, isoechoic, showing isoechoic to normal thyroid parenchyma, or hyperechoic, showing relatively more hyperechoic than adjacent normal thyroid parenchyma. Thyroid nodular vascularity was assessed and classified based on the standardized ultrasound report for thyroid nodules [10].

Internal content of the nodule was categorized according to the ratio of cystic to solid portion in the nodule and was classified as predominantly solid, $<50 \%$ cystic or predominantly cystic, $\geq 50 \%$ cystic. A spongiform appearance was defined as the aggregation of multiple micro-cystic components in more than $50 \%$ of the volume of the nodule. Margin of the nodule was categorized as welldefined margin or indistinct margin. Discontinuity of peripheral halo sign of the nodule was also evaluated.

Calcification was observed regarding the size of the nodule and was classified as microcalcification, tiny, punctuate echogenic foci of $1 \mathrm{~mm}$ or less either with or without posterior acoustic shadowing or macrocalcification, and punctuate echogenic foci larger than $1 \mathrm{~mm}$ in size.

\section{Statistical Analysis}

Statistical analysis was done by SPSS 17.0. Sensitivity (Se), specificity (Sp), positive predictive value (PPV), negative predictive value (NPV), and accuracy were estimated using following formulas (abbreviations: TP, true positive; FN, false negative; TN, true negative; FP, false positive).

$$
\begin{gathered}
S e=[T P /(T P+F N)] \times 100 \% \\
S P=[T N /(T N+F P)] \times 100 \% \\
P P V=[T P /(T P+F P)] \times 100 \% \\
N P V=[T N /(T N+F N)] \times 100 \% \\
\text { Accuracy }=[(T P+T N) /(\text { Positive }+ \text { Negative })] \times 100 \%
\end{gathered}
$$

The sensitivity, specificity, positive and negative predictive values, accuracy, and likelihood ratios ( $95 \%$ confidence intervals [CI]) of US, and pathology were determined for each site of involvement. Parametric and nonparametric continuous variables were compared using Student's t-test, and categorical variables were compared using the chi-square test, Fisher's exact test, the MacNemar test, or the $Z$ statistic as appropriate. $P$ values $<0.05$ were considered statistically significant.

\section{Results}

Baseline characteristics of study group have been given in Table 1.

There was a significant correlation between echogenicity and pathologic results, which mean more hyper-echogenicity leads to more benign condition $(\mathrm{P}=0.004)$. There was no significant correlation between the type of calcification and pathologic results $(P=0.270)$. Also there was no significant correlation between the marginal appearance and pathologic results $(P=0.860)$. There was a significant correlation between the length and width with pathologic results, which mean the shorter length and width lead to a more benign condition ( $\mathrm{P}=0.015, \mathrm{P}=0.040$, respectively). Besides, there was no significant correlation between vascularity and the age with pathologic results $(P=0.450, P=0.930$, respectively). Accuracy in evaluation benign and malignant involvement of thyroid nodules in our study was $72.96 \%$ and 93.49\% respectively (Table 2 ). 
Table 2. Statistical results of ultrasonography and pathology in detection of benign and malignant thyroid nodules

\begin{tabular}{|l|c|c|}
\hline \multicolumn{1}{|c|}{ Diagnosis } & Benign & Malignant \\
\hline True positive, no & 204 & 12 \\
True negative, no & 20 & 275 \\
False positive, no & 0 & 20 \\
False negative, no & 83 & 0 \\
Sensitivity, \% & 71.08 & 100 \\
Specificity, \% & 100 & 93.22 \\
Positive predictive value, \% & 100 & 37.50 \\
Negative predictive value, \% & 19.42 & 100 \\
Accuracy, \% & 72.96 & 93.49 \\
\hline
\end{tabular}

\section{Discussion}

Assessing the accuracy of US diagnosis of non-palpable benign and malignant nodules of the thyroid may lead promising findings, so the present study which aimed to observe the diagnostic accuracy US in assessment of thyroid masses in comparison with pathology, showed the accuracy of US in diagnosis benign and malignant thyroid nodules is acceptable.

Recently, Ghajarzadeh et al. determined the diagnostic accuracy of US in detecting malignant thyroid nodules, through a systematic review and meta-analysis of all available evidence. They claimed that US can be considered as a reliable screening tool for characterizing thyroid nodules, and detection of benign pathology in almost all cases, thus can be used to exclude many patients from further invasive assessments [11]. Zhuo et al. assessed the performance of acoustic radiation force impulse (ARFI) imaging to differentiate benign from malignant thyroid nodules in 182 patients who needed thyroid surgery. They reported that ARFI imaging may be helpful to differentiate benign thyroid nodules from malignant, and the selecting measurement position is important, as well as it has worthy diagnostic value in clinical applications [12]. Campanella et al. conducted a study to quantify the risk of malignancy of thyroid nodules using US by conducting a systematic review and meta-analysis of all available studies. They showed the highest risk of malignancy was found for nodule height greater than width, and microcalcifications [13]. Kim et al. investigated the benign thyroid nodules at initial fine-needle aspiration biopsy (FNAB) to determine the percentage of nodules that increased in volume by more than $50 \%$ as being an indicator of malignancy in concordance with long-term US, and showed that a positive FNAB result for malignancy is significantly more likely in the presence of suspicious US features [14]. Yang et al. explored the values of US in differentially diagnosing benign and malignant thyroid nodules, and showed that US can be used to evaluate the malignancy risk of thyroid nodules and help to make the right decision in clinics [15]. Wu et al. evaluated the diagnostic utility of conventional US in differentiating degenerating cystic thyroid nodules mimicking malignancy from thyroid carcinoma, and showed that US contributes to increasing the performance in differential diagnosis of thyroid nodule and malignancy [16].

Phuttharak et al. compared diagnostic performance of US and US plus color Doppler ultrasound in predicting malignancy of thyroid nodules by using tissue diagnosis as the reference standard, and reported that combination method findings improve the diagnosis of malignancy in thyroid nodules [9]. In contrast, Batawil et al. evaluated the predictive value of ultrasonography and the frequency of malignancy in patients who had indeterminate thyroid nodules, and showed that limited accuracy of US risk factors, surgical intervention is the treatment of choice for thyroid nodules [17]. Our result showed that US considerably can help in deterring benign thyroid nodule, and may provide more promising results in case of malignant thyroid nodules.

\section{Conclusion}

This study showed that due to the high incidence of thyroid nodules and the availability of noninvasive procedures such as US, this method can play a key role in differentiating benign from malignant thyroid nodules. Therefore, it is better that thyroid nodules, which are suspected to malignancy in the ultrasound undergo FNA-guided US. You must bear in mind that thyroid nodules which are suspected of malignancy in the ultrasound include, low echogenicity and nodule size of more than $10 \mathrm{~mm}$ in length and width. Other ultrasound findings such as microcalcifications, vascularity, size of the nodules and the age of the patient paly no major role in determining benign thyroid nodules from malignant.

This study showed that pathology and US have an incremental role in diagnosis of benign thyroid nodules from malignant. According to the high specificity and positive predictive value of US in diagnosing benign nodules and high accuracy in the differential diagnosis of malignance thyroid nodules from benign, merging these two methods (US and Pathology) in patients with thyroid nodules helps physicians in making decisions about treatment of the nodules.

Conflict of interest: none declared.

\section{References}

1. Wilhelm S. Evaluation of thyroid incidentaloma. Surg Clin North Am 2014; 94(3): 485-497. (doi: 10.1016/j.suc.2014.02.004) (PMID: 24857572)

2. Mackenzie EJ, Mortimer RH. 6: Thyroid nodules and thyroid cancer. Med J Aust 2004; 180(5): 242-247. (PMID: 14984346)

3. Kwak JY, Kim EK. Ultrasound elastography for thyroid nodules: recent advances. Ultrasonography 2014; 33(2): 75-82. (doi: 10.14366/usg.13025) (PMID: 24936499) (PMCID: PMC4058985)

4. Morgan JL, Serpell JW, Cheng MS. Fine-needle aspiration cytology of thyroid nodules: how useful is it? ANZ J Surg 2003; 73(7): 480-483. (PMID: 12864820) (doi: 10.1046/j.1445-1433.2003.02670.x)

5. Papini E, Guglielmi R, Bianchini A, Crescenzi A, Taccogna S, Nardi F, et al. Risk of malignancy in nonpalpable thyroid nodules: predictive value of ultrasound and color-Doppler features. J Clin Endocrinol Metab 2002; 87(5): 1941-1946. (doi: 10.1210/jcem.87.5.8504) (PMID: 11994321)

6. Soelberg KK, Bonnema SJ, Brix TH, Hegedus L. Risk of malignancy in thyroid incidentalomas detected by $18 \mathrm{~F}$-fluorodeoxyglucose positron emission tomography: a systematic review. Thyroid 2012; 22(9): 918 925. (doi: 10.1089/thy.2012.0005) (PMID: 22827552)

7. Lee HK, Hur MH, Ahn SM. Diagnosis of occult thyroid carcinoma by ultrasonography. Yonsei Med J 2003; 44(6): 1040-1044. (PMID: 14703614) (doi: 10.3349/ymj.2003.44.6.1040)

8. Lin JD, Huang BY, Chao TC, Hsueh C. Diagnosis of occult thyroid carcinoma by thyroid ultrasonography with fine needle aspiration cytology. Acta Cytol 1997; 41(6): 1751-1756. (PMID: 9390136)

9. Phuttharak W, Somboonporn C, Hongdomnern G. Diagnostic performance of gray-scale versus combined gray-scale with colour doppler ultrasonography in the diagnosis of malignancy in thyroid nodules. Asian Pac J Cancer Prev 2009; 10(5): 759-764. (PMID: 20104965) 
10. Andrioli M, Carzaniga C, Persani L. Standardized ultrasound report for thyroid nodules: the endocrinologist's viewpoint. European Thyroid Journal 2013; 2(1): 37-48. (doi: 10.1159/000347144) (PMID: 24783037) (PMCID: PMC3821499)

11. Ghajarzadeh M, Sodagari F, Shakiba M. Diagnostic accuracy of sonoelastography in detecting malignant thyroid nodules: a systematic review and meta-analysis. Am J Roentgenol 2014; 202(4): W379-W389. (doi: 10.2214/AJR.12.9785) (PMID: 24660737)

12. Zhuo J, Ma Z, Fu WJ, Liu SP. Differentiation of benign from malignant thyroid nodules with acoustic radiation force impulse technique. $\mathrm{Br} J$ Radiol 2014; 87(1035): 20130263. (doi: 10.1259/bjr.20130263) (PMID: 24588664) (PMCID: PMC4064611)

13. Campanella P, lanni F, Rota CA, Corsello SM, Pontecorvi A. Quantification of cancer risk of each clinical and ultrasonographic suspicious feature of thyroid nodules: a systematic review and metaanalysis. Eur J Endocrinol 2014; 170(5): R203-R211. (doi: 10.1530/EJE13-0995) (PMID: 24536085)

14. Kim SY, Han KH, Moon HJ, Kwak JY, Chung WY, Kim EK. Thyroid nodules with benign findings at cytologic examination: results of longterm follow-up with US. Radiology 2014; 271(1): 272-281. (doi: 10.1148/radiol.13131334) (PMID: 24475857)

15. Yang C, Han C, Wang LP, Feng N, Wang YF, You XD. Exploration on an ultrasonographic imaging reporting and data system in malignancy grading of thyroid nodules. Zhonghua Zhong Liu Za Zhi 2013; 35(10): 758-763. (PMID: 24378098)

16. Wu HX, Zhang BJ, Wang J, Zhu BL, Zang YP, Cao YL. Conventional ultrasonography and real time ultrasound elastography in the differential diagnosis of degenerating cystic thyroid nodules mimicking malignancy and papillary thyroid carcinomas. Asian Pac J Cancer Prev 2013; 14(2): 935-940. (PMID: 23621264)

17. Batawil N, Alkordy T. Ultrasonographic features associated with malignancy in cytologically indeterminate thyroid nodules. Eur J Surg Oncol 2014; 40(2): 182-186. (doi: 10.1016/j.ejso.2013.11.015) (PMID: 24373298)

Authors:

Morteza Tahmasebi - MD, Radiologist, Department of Radiology, Golestan Hospital, Ahvaz Jundishapur University of Medical Sciences, Ahvaz, Iran.

Mohammad Reza Boostani Dezfouli - MD, Radiologist, Department of Radiology, Golestan Hospital, Ahvaz Jundishapur University of Medical Sciences, Ahvaz, Iran.

Mohammad Momen Gharibvand - MD, Radiologist, Department of Radiology, Golestan Hospital, Ahvaz Jundishapur University of Medical Sciences, Ahvaz, Iran.

Alireza Jahanshahi - MD, Endocrinologist, Department of Endocrinology and Metabolism, Imam Khomeini Hospital, Ahvaz Jundishapur University of Medical Sciences, Ahvaz, Iran.

Neda Nikpour - MD, Radiologist, Department of Radiology, Golestan Hospital, Ahvaz Jundishapur University of Medical Sciences, Ahvaz, Iran.

Fakher Rahim - PhD in Molecular Medicine, Health research institute, Hearing research center, Ahvaz Jundishapur University of Medical Sciences, Ahvaz, Iran. 